\title{
Stochastic Optimal Control Model of Dengue Disease
}

\author{
K.L. Muruganantha Prasad, P.S. Stem Edilber, \\ S. Mookan
}

\section{INTRODUCTION}

The awareness that transmission dynamics of infectious diseases, the preventive and treatment strategies that are administered follow certain laws that could be modeled mathematically have been a beautiful child to the human conscience since the first incidence of epidemic outbreak [1].

The awakened consciousness that preventive and treatment strategies which are administered in the approach to finding solution to an epidemic outbreak require some mathematical calculations have contributed immensely in removing the subterfuge mist that has surrounded the elucidating of the transmission dynamics of infectious disease, the control strategies and the prevention of epidemic from invading a population of susceptible and has led us out into the light of strategic epidemiological control measures [2].

The mathematical computations which have been involved in the study of the dynamics of infectious diseases have brought a great surge of excitement in the hearts of modelers, governments, public health workers and all stakeholders as it has created awareness with regards to transmission dynamics and control measures of infectious diseases [3].

In this research article, we consider the dengue model with standard incidence rate as proposed by [9], as studied in section 2 . The section 3 deduces an optimal control problem that assesses the impact of some control strategies: prevention, treatment and application of insecticide spray on the vector by incorporating time dependent control functions. The necessary conditions for an optimal and the corresponding states are then derived by employing the Pontryagin's Maximum Principle. Finally, in section 4, the resulting optimality system is numerically solved and computed to investigate the optimum control strategy that would be efficacious to be implemented in reducing the

Revised Manuscript received July 10, 2019.

K.L. Muruganantha Prasad, Department of Mathematics, H.H. The Rajahs College, Pudhukottai, Tamilnadu, India.

P.S. Stem Edilber, Research Scholar, H.H. The Rajahs College, Pudhukottai, Tamilnadu, India.

S. Mookan, Department of Mathematics, PET Engineering College, Valliyoor, Tamilnadu, India.

\begin{abstract}
In this research article, an optimal control model of dengue disease with standard incidence rate is proposed. Maximum Principle was employed to derive the necessary conditions for the existence of optimal control. Stochastic version of the model is derived by introducing a random perturbation in the main parameters of the model equations. Numerical solution of the optimality was derived and computed to investigate the optimum control strategy that would be efficacious to be implemented in reducing the number of exposed and infected humans as well as illustrating the explicit differences in the dynamics of the models.
\end{abstract}

number of exposed and infected humans. Further, in section 6-7, Stochastic version of system (1) is obtained by introducing a random perturbation to the equations of the systems. In section 8, stochastic numerical method-EulerMaruyama is discussed. Section 9 gives numerical examples to show the dynamics of the deterministic, control and the stochastic systems, and to illustrate the differences in the dynamics of the models.

\section{MODEL FRAMEWORK}

This section presents a dengue model with standard incidence rate. The human population at time $t$ is categorized into four classes: susceptible human $\mathrm{SH}(\mathrm{t})$, exposed human $\mathrm{EH}(\mathrm{t})$, infectious human $\mathrm{IH}(\mathrm{t})$, recovered human $\mathrm{RH}(\mathrm{t})$, respectively. The mosquito population is divided into three classes: susceptible mosquito $\mathrm{Sv}(\mathrm{t})$, exposed mosquito $\operatorname{Ev}(\mathrm{t})$ and infectious mosquito $\operatorname{Iv}(\mathrm{t})$, respectively. The total populations of human and mosquito at any time are given by

$\mathrm{NH}(\mathrm{t})=\mathrm{SH}(\mathrm{t})+\mathrm{EH}(\mathrm{t})+\mathrm{IH}(\mathrm{t})+\mathrm{RH}(\mathrm{t})$ and $\mathrm{Nv}(\mathrm{t})=\mathrm{Sv}(\mathrm{t})+$ $\operatorname{Ev}(\mathrm{t})+\operatorname{Iv}(\mathrm{t})$ respectively.

The mosquito population does not include immune class as mosquitoes never recover from infection, that is, their infective period ends with their death due to their short lifecycle. Further, it is accepted that the recovered human have some level of immunity to the disease and do not get clinically ill, but still harbor low levels of parasite in their blood streams which are transmitted to a susceptible mosquitoes during bites [9]. The mathematical differential equations of the dynamics as proposed by Pariyaporn et al [9] are:

$$
\begin{gathered}
\frac{d S_{H}}{d t}=\pi_{H}+\rho_{H} R_{H}-\sigma_{v} \beta_{H} \frac{I_{v}}{N_{H}} S_{H}-\mu_{H} S_{H} \\
\frac{d E_{H}}{d t}=\sigma_{v} \beta_{H} \frac{I_{v}}{N_{H}} S_{H}-\left(v_{H}+\mu_{H}\right) E_{H} \\
\frac{d I_{H}}{d t}=v_{H} E_{H}-\left(\gamma_{H}+\mu_{H}+\delta_{H}\right) I_{H} \\
\frac{d R_{H}}{d t}=\gamma_{H} I_{H}-\left(\rho_{H}+\mu_{H}\right) R_{H} \ldots \ldots \ldots \ldots \ldots \ldots \\
\frac{d S_{v}}{d t}=\pi_{v}-\sigma_{v}\left(\frac{\beta_{V} I_{H}+\beta_{V H} R_{H}}{N_{H}}\right) S_{V}-\mu_{V} S_{V} \\
\frac{d E_{V}}{d t}=\sigma_{V}\left(\frac{\beta_{V} I_{H}+\beta_{V H} R_{H}}{N_{H}}\right) S_{V}-\left(V_{v}+\mu_{V}\right) E_{V} \\
\frac{d I_{v}}{d t}=V_{v} E_{v}-\mu_{V}-\delta_{1} \omega_{3} I_{V}
\end{gathered}
$$

The model assumed that people enter the susceptible class either through bite or immigration at a recruitment rate $\sigma_{v} \beta_{H} \frac{I_{v}}{N_{H}}$.

Published By:

Blue Eyes Intelligence Engineering 
When an infectious mosquito bites a susceptible human, there is some finite probability that the dengue parasite will be passed on to the human at the rate of $\sigma_{v} \beta_{H} \frac{I_{v}}{N_{H}}$, and the person will move to the exposed class. Individuals from the exposed class enter the infectious class at a rate, that is the reciprocal of the duration of the latent period. Recovered infectious humans move to the recovery class at a rate and die from the infection at a rate. It is assumed that recovered individuals have temporary immunity that can be lost and are again susceptible to re infection at a rate $\rho_{H}$. All human classes leave the population through the same natural death rate $\mu_{H}$. The infectious human leaves the population at a per capita disease-induced death rate. For the mosquito population, susceptible mosquitoes are recruited by birth at a constant rate, independent of the actual number of adult mosquitoes. This assumption reflects reality, since only a fraction of a large reservoir of eggs and larvae matures to the adult stage, and this process does not depend directly on the size of the adult mosquito population. All mosquito populations die due to their finite life span at the same rate of. Susceptible mosquitoes are infected by biting the infectious human and recovered human at the infection rate $\sigma_{V}\left(\frac{\beta_{V} I_{H}+\beta_{V H} R_{H}}{N_{H}}\right)$. Susceptible mosquitoes which are infected move to the exposed mosquito class and progress to the infectious mosquito class at a rate $V_{v}$.

\section{OPTIMAL CONTROL STRATEGIES}

In this section, the state system (1) is modified to assess the impact of some control strategies: prevention, treatment and application of insecticide spray on vector. In the human population, the associated force of infection is reduced by a factor of $\left(1-\omega_{1}(t)\right)$ where $\omega_{1}(t)$ represents the use of preventive control of sleeping under treated mosquito net to prevent direct contact and bite from infected mosquito.

$\omega_{2}(t)$ represents an application of insecticide spray on the bleeding grounds of the vector. It is undeniable fact that mosquito bleeds and flourish under a favorable climate conditions such as rainy seasons and hot climatic conditions . Therefore, strenuous effort is required in application of insecticide at the bleeding fields of the vector at these times. Thus, the reproduction rate of the mosquito population is reduced by a factor of $\left(1-\omega_{2}(t)\right)$.

The control function $\omega_{3}(t)$ measures the rate at which infected individuals are treated at each time with dengue drugs for the control of the disease. We assume that $\omega_{3} I_{H}$ individuals at any time $(\mathrm{t})$ are removed from the infective class and added to the removed class.

Further, we assume that the mortality rate of the vector population increases at a rate proportional $\delta_{1} \omega_{3} I_{V}(t)$, where $\delta_{1}>0$ is a constant rate. With regards to these underlying assumptions, an optimal control model for malaria disease is formulated that deduces prevention and treatment strategies with a minimal cost of implementation. Hence, the dynamics of system (1) are modified to the following system of equations:

$$
\begin{gathered}
\frac{d S_{H}}{d t}=\pi_{H}+\rho_{H} R_{H}-\left(1-\omega_{1}\right) \sigma_{v} \beta_{H} \frac{I_{v}}{N_{H}} S_{H}-\mu_{H} S_{H} \\
\frac{d E_{H}}{d t}=\left(1-\omega_{1}\right) \sigma_{v} \beta_{H} \frac{I_{v}}{N_{H}} S_{H}-\left(v_{H}+\mu_{H}\right) E_{H}
\end{gathered}
$$

$$
\begin{gathered}
\frac{d I_{H}}{d t}=v_{H} E_{H}-\left(\gamma_{H}+\mu_{H}+\delta_{H}+\omega_{3}\right) I_{H} \\
\frac{d R_{H}}{d t}=\gamma_{H} I_{H}-\left(1-\omega_{1}\right)\left(\rho_{H}+\mu_{H}\right) R_{H} \ldots \ldots \ldots \ldots \ldots \ldots \ldots \\
\frac{d S_{v}}{d t}=\pi_{v}-\left(1-\omega_{2}\right) \sigma_{v}\left(\frac{\beta_{V} I_{H}+\beta_{V H} R_{H}}{N_{H}}\right) S_{V}-\mu_{V} S_{V} \\
\frac{d E_{V}}{d t}=\left(1-\omega_{2}\right) \sigma_{V}\left(\frac{\beta_{V} I_{H}+\beta_{V H} R_{H}}{N_{H}}\right) S_{V}-\left(V_{v}+\mu_{V}\right) E_{V} \\
\frac{d I_{v}}{d t}=V_{v} E_{v}-\mu_{V}-\delta_{1} \omega_{3} I_{V}
\end{gathered}
$$

and infected human individuals and the infected vector population and maximizes the total number of recovered individuals through preventive and treatment strategies, by employing feasible minimal time dependent control variables . $\omega_{1}(t), \omega_{2}(t), \omega_{3}(t)$ respectively.

$$
\begin{gathered}
\mathrm{J}\left(\omega_{1}, \omega_{2}, \omega_{3}\right)= \\
\int_{t_{v}}^{t_{f}}\left(A_{1} E_{H}+A_{2} I_{H}+A_{3} I_{v}+\right. \\
12 a 1 w 12+a 2 w 22+a 3 w 32 d t \ldots \ldots \ldots . . .
\end{gathered}
$$

With appropriate initial conditions, we consider an optimal control problem to minimize the objective functional given by the quantities $A 1, A 2$ and $A 3$ denote the weight constant of the exposed and infected human individuals and the infected vector population. Again, the quantities $w 1, w 2$ and $w 3$ are weight constants for minimizing the number of exposed and infected human individuals and treatment of infected human individuals Further, the terms $1 / 2 a_{1} w_{1}^{2}, 1 / 2 a_{2} w_{2}^{2} 1 / 2 a_{3} w_{3}^{2}$ represent the cost associated with the minimizing the exposed and infected and treatment of infected human individuals.

We choose a quadratic cost on the controls as a reflection of what is in other literature on epidemic control models Here, we seekan optimal control $w_{1}^{*}, w_{2}^{*}, w_{3}^{*}$ such that

$$
\begin{gathered}
J\left(w_{1}^{*}, w_{2}^{*}, w_{3}^{*}\right)=\min \left\{J \left(w_{1}, w_{2}, w_{3} / w_{1}, w_{2}, w_{3} \in\right.\right. \\
W\} \ldots \ldots \ldots \ldots \ldots . . .(4)
\end{gathered}
$$

Where $\left\{w_{1}, w_{2}, w_{3} / w_{i}(\mathrm{t})\right.$ is lebesgue measurable with

$$
\left.0 \leq w_{i}(\mathrm{t}) \leq 1, i=1,2,3\right\} \ldots \ldots(5)
$$

Applying the Pontryagin's Maximum Principle [25], the system (2) and (3) are converted into minimizing the Hamiltonian $H$, with respect to $w 1, w 2$ and $w 3$

Where $H\left(S_{H}, E_{H}, R_{H}, S_{V}, E_{V}, I_{V}, W, \lambda_{1}, \lambda_{2}, \lambda_{3}, \lambda_{4}, \lambda_{5}, \lambda_{6}, \lambda_{7}, t\right)$ $=A_{1} E_{H}+A_{2} I_{H}+$ $A_{3} I_{v}+1 / 2 a_{1} w_{1}^{2}+1 / 2 a_{2} w_{2}^{2}+\frac{1}{2 a_{3} w_{3}^{2}}+\lambda_{1} \frac{d S_{H}}{d t}+\lambda_{2} \frac{d E_{H}}{d t}+$ $\lambda_{3} \frac{d I_{H}}{d t}+\lambda_{4} \frac{d R_{H}}{d t}+\lambda_{5} \frac{d S_{V}}{d t}+\lambda_{6} \frac{d E_{V}}{d t}+\lambda_{7} \frac{d I_{V}}{d t}$

Where $\lambda_{i}$ for $\mathrm{i}=1, \ldots \ldots \ldots \ldots, 7$ are adjoint variables to be determined

\section{THEOREM}

There exist an optimal control $\mathrm{W}=\left\{w_{1}^{*}, w_{2}^{*}, w_{3}^{*}\right\} \in W$ such that

$J\left(w_{1}^{*}, w_{2}^{*}, w_{3}^{*}\right)=\min _{(w 1, w 2, w 3) \in W} J\left(w_{1}, w_{2}, w_{3}\right)$ subject to control system (2) with initial conditions at $\mathrm{t}=0$.

Furthermore optimal control $w_{1}^{*}, w_{2}^{*}, w_{3}^{*}$ are given by

$$
\begin{aligned}
& w_{1}^{*}=\max \left\{\min \left\{\frac{\sigma_{v} \beta_{v}\left(\lambda_{2}-\lambda_{1}\right) S_{H}^{*} I_{v}^{*}}{a_{1} N_{H}}, 1\right\} 0\right\} \\
& \text { Published By: } \\
& \text { Blue Eyes Intelligence Engineering Exploring innov } \\
& \text { \& Sciences Publication }
\end{aligned}
$$




$$
\begin{gathered}
w_{2}^{*}=\max \left\{\min \left\{\frac{\sigma_{v}\left(\lambda_{6}-\lambda_{5}\right)\left(\beta_{v} I_{H}^{*}+\beta_{v H} R_{H}^{*}\right) S_{V}^{*}}{a_{1} N_{H}}, 1\right\}, 0\right\} \\
w_{3}^{*}=\max \left\{\min \left\{\lambda_{7} \delta_{1} I_{V}^{*}+\left(\lambda_{3}-\lambda_{4}\right) I_{H}^{*}, 1\right\}, 0\right\}(12) \\
\text { V. NUMERICAL EXAMPLES AND } \\
\text { DISCUSSION \& RESULTS }
\end{gathered}
$$

\section{NUMERICAL EXAMPLES AND DISCUSSION \& RESULTS}

In this section, we asses by means of numerical approach the effect of the control strategies on the transmission dynamics of dengue model.

In order to achieve this, an iterative scheme is applied in solving the optimality system: state system and adjoint system.

First, the state system of equations is solved with arbitrary guess for the controls over a simulated time frame by employing fourth order Runge-kutta scheme. As a result of the presence of boundary conditions, the adjoint system is solved by backwards fourth order Runge-kutta using the immediate iterative solutions of the state equation. The controls are updated by means of a convex combination of the previous controls as well as the characterizations (10), (11) and (12).

The entire process is repeated until the values of the unknowns at the previous itearations are closed to the one at the current iteration.

The models assess the impact of control strategies on the transmission dynamics of Malaria disease with standard incidence rate. We investigate the effect of control strategies: prevention, treatment and application of insecticide spray on vector. This is done by numerically simulating the results of the dynamics of the disease to ascertain the stated scenarios of the strategies with parameter values $S_{H}=950000 E_{H}=500000 I_{H}=25000$ $R_{H=10 S_{V}}=850000 E_{V}=300000$

$I_{V}=850000 \quad a_{1}=500 \quad a_{2}=30 \quad a_{3}=10 A_{1}=10$ $A_{2}=10$ and $A_{3}=10$ from (9).

We further assumed that the weight factor, a1, associated with control w1 is greater than A1, A2, A3, a2 and a3 respectively, which are association of controls w2 and w3 . This holds on the ground that the cost of implementing w1 includes, educational campaign programs in our public institutions such as schools, health centres and churches and media advertisements such as Television and radio educational campaigns to educate the public on the use of mosquito nets and the danger of exposing oneself to the mercy of the vector especially in pregnant women and infants.

The cost of applying insecticide spray includes the insecticide cost and labour cost while treatment cost includes hospitalization, medical examination and the administration of dengue drugs in the infected persons. The parameter values used in the simulations are estimated based on the dengue disease as provided in Table 1. Other parameters were chosen arbitrary for the purpose of the

\begin{tabular}{|c|c|c|}
\hline parameter & Description & $\begin{array}{l}\text { Estimated } \\
\text { value }\end{array}$ \\
\hline$\pi_{H}$ & $\begin{array}{l}\text { Recruitment rate of } \\
\text { human }\end{array}$ & 2.5 \\
\hline$\beta_{H}$ & $\begin{array}{l}\text { Transmission probability } \\
\text { from an infectious } \\
\text { mosquito to a } \\
\text { susceptible human }\end{array}$ & 0.9 \\
\hline$\beta_{v}$ & $\begin{array}{l}\text { Transmission probability } \\
\text { from an infectious } \\
\text { human to a susceptible } \\
\text { mosquito }\end{array}$ & 0.8 \\
\hline$\beta_{v H}$ & $\begin{array}{l}\text { Transmission probability } \\
\text { from an recovered } \\
\text { human to a susceptible }\end{array}$ & 0.009 \\
\hline$\mu_{H}$ & $\begin{array}{l}\text { Natural death rate } \\
\text { human }\end{array}$ & 0.004 \\
\hline$\delta_{H}$ & $\begin{array}{l}\text { Death rate from the } \\
\text { disease }\end{array}$ & 0.00354 \\
\hline$\gamma_{H}$ & $\begin{array}{ll}\text { Infectious } & \text { human } \\
\text { recovery rate }\end{array}$ & 0.003704 \\
\hline$V_{H}$ & $\begin{array}{l}\text { Progression rate from } \\
\text { EH to I H class }\end{array}$ & 0.08333 \\
\hline$V_{v}$ & $\begin{array}{l}\text { Progression rate from } \\
\text { EV to Ivclass }\end{array}$ & 0.1 \\
\hline$\rho_{H}$ & $\begin{array}{l}\text { Rate of loss immunity in } \\
\text { human }\end{array}$ & 0.0146 \\
\hline$\pi_{v}$ & $\begin{array}{l}\begin{array}{l}\text { Recruitment rate of } \\
\text { mosquitoes }\end{array} \\
\end{array}$ & 500 \\
\hline$\sigma_{v}$ & Biting rate of vector & 2.9 \\
\hline$\mu_{v}$ & $\begin{array}{l}\text { Natural death rate of } \\
\text { vector }\end{array}$ & 0.0714 \\
\hline$\alpha_{H}, \alpha_{v}$ & $\begin{array}{l}\text { The level at which the } \\
\text { force of infection } \\
\text { saturates }\end{array}$ & $(0,1)$ \\
\hline
\end{tabular}
numerical simulation.

\section{CONCLUSION}

In this research article, an optimal control model of dengue disease with standard incidence rate was mathematically formulated to study the transmission dynamics of the disease in order to analyze the optimum control strategy that would be efficacious to be implemented to control the disease at a minimal cost. Three control functions were introduced to assess and measure empirically the efficacy of the use of sleeping under treated mosquito net to prevent direct contact and bite from infected mosquito, the application of insecticide spray on the bleeding grounds of the vector and the rate at which infected humans are treated at each time of the infection. The analysis proved that the optimal control strategies considered have an optimum and incomparable results on the reduction of the number of exposed and infected humans and mosquitoes as compared to the model without control . The numerical examples depict that despite the standard incidence rate, the proposed strategies are effective in the reduction of the number of the exposed and infected human and mosquito of the disease. 


\section{REFERENCES}

1. Murray JD. Mathematical Biology I. Spriger-Verlag Berlin Heidelbery; 2002.

2. Brauer F, Van den Driessche P, Wu (Eds). Mathematical Epidemiology: Mathematical Biosciences subseries. SpringerVerlag Berlin Heidelberg. 2008.

3. Murray JD. Mathematical Biology I, Spriger-Verlag Berlin Heidelbery; 2003.

4. Global Technical Strategy for Malaria 2016-2030. WHO. 2015; Geneva: World Health Organization (WHO), Switzerland

5. . 5. Roll Back Malaria Partnership. Action and investment to defeat malaria 2016-2030. For a Malaria free World. 2015; Geneva: World Health Organization (WHO), Switzerland.

6. Wei H, Li XZ, Martcheva M. An epidemic model of a vector-borne disease with direct transmission and time delay. J Math Anal Appl. 2008; 342: 895-908.

7. Abdullahi MB, Hasan YA, Abdullah FA. A mathematical model of Malaria and the Effectiveness of Drugs. Appl Math Sci. 2013; 7: 3079-3095.

8. Mandal S, Sarkar RR, Sinha S. Mathematical models of malaria -a review. Malar J. 2011; 10: 202.

9. Pariyaporn RO, Chinviriyasit $\mathrm{W}$, Chinviriyasit S. The effect of incidence function in backward bifurcation for malaria model with temporary immunity. Math Biosci. 2015; 265: 47-64.

10. Feng X, Ruan S, Teng Z, Wang K. Stability and backward bifurcation in a malaria transmission model with applications to the control of malaria in China. Math Biosci. 2015; 266: 52- 64 . 